\title{
Orientações recebidas do serviço de saúde por pacientes para o tratamento do portador de diabetes mellitus tipo 2
}

\author{
Advices received from health service by patients for the \\ treatment of type 2 diabetes mellitus
}

Fernanda Pontin de Mattos GUIMARÃES 1

Angela Maria Magosso TAKAYANAGUI ${ }^{2}$

RE S U M O

O Diabetes Mellitus é uma doença crônica que está afetando a população de forma crescente, tornando-se um sério problema de Saúde Pública. O objetivo deste estudo foi realizar um levantamento das principais orientações recebidas por um grupo de pacientes diabéticos, no momento do diagnóstico. Foram entrevistados 29 indivíduos inseridos no Programa de Assistência ao Diabético de uma Unidade Básica e Distrital de Saúde de Ribeirão Preto, SP. As orientações recebidas para o tratamento da doença, no momento do diagnóstico, foram fornecidas, em sua maioria, por profissional médico (96,5\%), indicando a ausência de outras categorias profissionais. Destas prescrições mencionadas, apenas 17,2\% abrangiam as recomendações da Sociedade Brasileira de Diabetes, incluindo informações sobre dieta, exercício físico e uso de medicamento. Por outro lado, $82,8 \%$ dos pacientes mencionaram ter sido orientados apenas para o tratamento com dieta e/ou medicação, sem qualquer instrução para a prática da atividade física. Pelos resultados, pode-se concluir a necessidade de as equipes de saúde reverem suas práticas de educação em saúde no tratamento do Diabetes Mellitus tipo 2, valorizando também as orientações relativas a mudanças de estilo de vida dos pacientes. Além disso, há de se destacar a importância de uma equipe de saúde multiprofissional, visando uma melhor qualidade da assistência prestada.

Termos de indexação: diabetes mellitus, orientação, saúde pública.

A B S T R A C T

Diabetes Mellitus is a chronic-degenerative disease which is affecting the population increasingly, becoming a serious public health problem. In this study, it was carried out a survey of the main advice's received, at the

\footnotetext{
1 Pós-Graduanda, Departamento de Enfermagem Materno-Infantil e Saúde Pública, Escola de Enfermagem de Ribeirão Preto, Universidade de São Paulo. Rua Otávio Magalhães, 89, Alto da Boa Vista, 14025-380, Ribeirão Preto, SP, Brasil. Correspondência para/Correspondence to: F.P.M. GUIMARÃES. E-mail: ferpontin@hotmail.com

2 Departamento de Enfermagem Materno-Infantil e Saúde Pública, Escola de Enfermagem de Ribeirão Preto, Universidade de São Paulo.E-mail: ammtakay@eerp.usp.br
} 
time of diagnosis, by a group of diabetic patients. Twenty-nine individuals, from the "Program of Assistance to Diabetic of a Basic and District Unit of Health of Ribeirão Preto, SP", were interviewed. The majority of the advices received at the time of diagnosis (96.5\%) for the treatment of the disease, had only been given by the physicians, indicating the absence of other kind of health care professional in this activity. Only $17.2 \%$ of these prescriptions contemplated specific and complete orientations, including dietary, physical exercise and medications, and following the recommendations of the Brazilian Diabetes Society. On the other hand, $82.8 \%$ of the interviewed patients had received only specific guidance on pharmacological treatment associated or not with dietary, both of them without instructions on physical exercise. From these results, it can be concluded that the health care teams should review their own practices concerning the health education on type 2 Diabetes Mellitus treatment, focusing on the orientations towards patients change in lifestyle. Furthermore, it is important to have multiprofessional health team, aiming to improve the quality of the health care given to these patients.

Index terms: diabetes mellitus, orientation, public health.

\section{N T R O D U ÇÃ O}

No Brasil, assim como em muitas outras localidades, o Diabetes Mellitus está sendo reconhecido como um importante problema de saúde pública, principalmente nos países em desenvolvimento, onde tem ocupado um percentual de 30 a 40\% das causas de morbidade entre adultos (Hoett, 1991). Esta doença também vem alcançando um espaço cada vez maior na literatura, com o aumento de pesquisas nesta área, representando, assim, uma doença crônica de grande importância.

A partir de um estudo multicêntrico sobre a prevalência do Diabetes Mellitus, realizado em nove capitais brasileiras, de 1986 a 1988, foi possível detectar que cerca de 7,6\% da população adulta é portadora de diabetes e, deste percentual, cerca de $50 \%$ desconhece esta condição. Este mesmo levantamento indicou uma predominância de 9,6\% de diabéticos entre os habitantes do município de São Paulo. Estes dados podem ser comparados à incidência em países desenvolvidos, onde esta doença é considerada um problema de saúde pública (Banco Mundial..., 1991).

Em termos de morbidade, o Diabetes Mellitus atualmente representa uma das principais doenças crônicas que afetam o homem contemporâneo, acometendo indivíduos de países em todos os estágios de desenvolvimento econômico-social (Pupo, 1989; Malerbi, 1991; Rull et al., 1992). Sua importância nas últimas décadas vem crescendo em decorrência de vários fatores, tais como: maior taxa de urbanização, aumento da expectativa de vida, industrialização, dietas hipercalóricas e ricas em hidratos de carbono de absorção rápida, deslocamento de populações para zonas urbanas, mudanças de estilo de vida, inatividade física e obesidade (São Paulo..., 1991).

Esta situação demonstra a necessidade de os serviços de saúde pública reverem suas práticas, com a implantação de ações para estabelecer medidas de prevenção e controle desta doença, com o objetivo de reduzir os índices de morbi-mortalidade. Neste mesmo sentido, as orientações do Ministério da Saúde (Brasil..., 1988) são para se efetuar intervenções em saúde antes da manifestação dos fenômenos patológicos. Assim, são recomendadas medidas preventivas, extrapolando-se as ações assistenciais e demandando-se práticas de saúde mais abrangentes para a população, a fim de minimizar o aparecimento dos fatores de risco ou reduzir a oportunidade de exposição das pessoas a eles. 
Segundo Lalonde citado por Dever (1988), a saúde é determinada por uma variedade de fatores, os quais o autor denomina de "Campo de Saúde", podendo ser agrupados em quatro grandes categorias: biologia humana, estilo de vida, ambiente e organização dos serviços de saúde. Este conceito de "campo de saúde" tem uma estrutura abrangente, permitindo uma ampla análise do binômio saúde-doença e uma investigação de seus determinantes.

Para este autor, a biologia humana é um elemento definido pela caracterização biológica e orgânica dos indivíduos, de modo que a herança genética de uma pessoa pode criar desordens genéticas, malformações congênitas ou retardamento mental. Além disso, o processo de maturidade e envelhecimento contribui para o surgimento de artrite, diabetes, aterosclerose e câncer.

Quanto ao estilo de vida, este segmento no modelo epidemiológico envolve o conjunto de decisões tomadas pelos indivíduos e que afetam sua própria saúde e sobre as quais eles têm maior ou menor controle (Dever, 1988). Pode ser dividido em atividades de lazer, padrões de consumo e riscos ocupacionais.

Com relação ao ambiente, trata-se de um fator definido como um evento externo ao corpo, sobre o qual o indivíduo tem pouco ou nenhum controle, como por exemplo: riscos à saúde provenientes da poluição sonora, do ar e da água.

Finalmente, como quarta categoria na classificação deste mesmo autor, encontra-se a organização dos serviços de saúde, dividida em três elementos: de prevenção, de cura, e de recuperação. Ela engloba disponibilidade, qualidade e quantidade de recursos para oferecer cuidados à saúde da população.

A partir deste conceito de "campo de saúde", pode-se elaborar um planejamento de ações específicas, apoiadas em cada uma das categorias mencionadas acima e voltadas para a promoção da saúde de indivíduos portadores de diabetes, bem como para a população em geral.
Embora na literatura existam vários conceitos de diabetes (Foss et al., 1989; Brasil..., 1993; Sociedade Brasileira..., 1997), segundo a recente publicação do Comitê Internacional de Especialistas, que trabalhou sob o patrocínio da Associação Americana de Diabetes, Diabetes Mellitus foi definido 'como um grupo de doenças metabólicas caracterizado por uma hiperglicemia resultante de defeitos na secreção de insulina, na ação da insulina ou em ambas' (Expert Committee..., 1999). Este quadro de hiperglicemia crônica no diabetes está associado, após longos períodos, ao prejuízo e à falência de vários órgãos, especialmente olhos, rins, nervos, coração e vasos sangüíneos.

Segundo a Sociedade Brasileira... (2000), a classificação atualmente recomendada incorpora o conceito de estágios clínicos do diabetes, desde a normalidade, passando pela tolerância à glicose diminuída e/ou glicemia de jejum alterada, até o diabetes mellitus propriamente dito. A nova classificação baseia-se na etiologia do diabetes: Tipo 1 - destruição da célula beta com deficiência absoluta de insulina; Tipo 2: varia entre a resistência insulínica e um defeito secretório; Outros tipos específicos: decorrentes de defeitos genéticos e de doenças ou induzidos por fármacos e agentes químicos; e diabetes gestacional: casos detectados na gravidez.

O tratamento do diabetes visa, predominantemente, o controle glicêmico. A Sociedade Brasileira de Diabetes (Programa..., 1996) preconiza também como objetivos: aliviar os sintomas, melhorar a qualidade de vida, prevenir complicações agudas e crônicas, reduzir a mortalidade e tratar as doenças associadas.

O tratamento básico e o controle da doença dos tipos de diabetes 1 e 2 consistem, primordialmente, de uma dieta específica, de atividade física e do uso adequado da medicação (antidiabéticos orais e/ou insulina). O resultado é obtido através de uma educação específica, com a necessidade de adoção pelos portadores de diabetes de determinadas medidas e práticas comportamentais. 
Segundo Chacra \& Lerário (1998), os estádios que envolvem o tratamento do indivíduo diabético tipo 2 são: Estádio I: tratamento dietético, programa de exercícios, mudança de estilo de vida, treinamento em automonitorização; Estádio II: uso de drogas antiobesidade e antidiabéticos orais em monoterapia ou em combinação, além das orientações do Estádio l; Estádio III: uso de insulina em tratamento oral ou instituição definitiva da insulina como monoterapia, além das orientações do Estádio l; Estádio IV: intensificação do tratamento insulínico com as orientações do Estádio I.

Segundo estes autores, o tratamento inicial consiste exclusivamente em uma mudança no estilo de vida, incluindo basicamente, a adaptação a um plano alimentar específico e a prática de atividade física. Concomitantemente, deve-se iniciar um programa de educação em diabetes, abordando temas de importância e dando ênfase ao plano alimentar e aos benefícios da atividade física.

Vários autores enfatizam a importância de se estimular de forma racional, nos indivíduos portadores de diabetes, a prática do exercício físico como parte do seu tratamento (Thurm \& Harper, 1992; Gordon, 1997). Mendes (1996) cita a importância do exercício físico e da mudança do estilo de vida em indivíduos idosos de uma comunidade de Ribeirão Preto - SP, incluindo portadores de doenças crônicas, entre elas o diabetes.

Atualmente tem havido uma tendência para a valorização de medidas não convencionais de assistência aos pacientes com doenças crônicas, ou seja, as centradas no tratamento não medicamentoso (Regenga et al., 1998; Boog \& Magrini, 1999), destacando-se a prática contínua e muitas vezes diária de exercícios físicos e de caminhadas. Incentiva-se também a valorização da alimentação adequada, a qual passa a ser vista mais como um benefício do que como um castigo.

Seguindo esta tendência, além de outras iniciativas, Ribeirão Preto conta com o Programa de Integração Comunitária (PIC), cujo objetivo é incentivar os portadores de doenças crônicas à prática da atividade física; este programa inclusive está incorporado às ações assistenciais da Secretaria Municipal de Saúde. São desenvolvidas também atividades de abrangência coletiva relacionadas à assistência comunitária, promoção e educação em saúde.

No entanto, este município possui um modelo assistencial praticado nos serviços de saúde ainda com predomínio da intervenção curativa centrada na assistência médica, com conseqüente utilização de tecnologias de maior custo.

O atendimento, nesta cidade, ao portador de diabetes inserido no Programa de Assistência ao Indivíduo Diabético ocorre através de um agendamento na própria Unidade Básica e Distrital de Saúde (UBDS) mais próxima de sua residência e enquadrada no programa. O médico, quando necessário, encaminha o paciente, após consulta, para receber orientações dos auxiliares de enfermagem quanto à dieta e à medicação. Para a orientação dietética é fornecido um plano alimentar já estabelecido pelo serviço.

Neste contexto, o objetivo deste trabalho foi conhecer o tipo de orientação recebida por um grupo de portadores de diabetes atendidos em uma Unidade Básica e Distrital de Saúde da Secretaria Municipal de Saúde de Ribeirão Preto, tomando-se como hipótese que o indivíduo, quando portador de uma doença crônica como o diabetes centraliza seu tratamento no profissional médico e na medicação, dando pouca importância a outros tipos de recomendações, principalmente quando fornecidas por outros profissionais de saúde.

\section{CASUÍSTICA E MÉTODOS}

Esta investigação foi realizada por meio de um levantamento de dados junto a indivíduos portadores de diabetes mellitus tipo 2 inscritos no Programa de Assistência aos Indivíduos Diabéticos da Unidade Básica e Distrital de Saúde Doutor 
Ítalo Baruffi da Secretaria Municipal de Saúde de Ribeirão Preto, SP. Segundo Rigsby (1987), esta estratégia metodológica permite identificar a incidência e a distribuição de particularidades ou relações entre características de uma determinada população. Inicialmente não foi definido o número de participantes para o estudo e sim o período para realização da coleta de dados, de fevereiro de 1999 a junho do mesmo ano. A amostra, portanto, foi constituída de 29 indivíduos que atenderam aos critérios de inclusão para participação nesta pesquisa, a saber: ter diagnóstico de diabetes tipo 2, estar em tratamento há pelo menos 6 meses, ter idade superior a 30 anos, ser residente na área de abrangência da UBDS e dar a anuência para participar do estudo.

O instrumento utilizado foi um questionário com perguntas semi-estruturadas, abrangendo a identificação do indivíduo, tempo e local do diagnóstico do diabetes, tipo de orientação recebida neste momento e categoria profissional de quem o orientou pela primeira vez.

Os indivíduos foram selecionados aleatoriamente e entrevistados no dia do seu comparecimento ao serviço para consulta médica previamente agendada.

\section{RESULTADOSE DISCUSS ÃO}

Durante o período proposto, participaram desta pesquisa 29 indivíduos, sendo $31 \%$ do sexo masculino e $69 \%$ do sexo feminino, constituindo, portanto, uma amostra de dois indivíduos do sexo feminino para cada sujeito do sexo masculino.

Pôde-se observar na distribuição por faixa etária desta população diabética, que 58,6\% eram indivíduos acima de 60 anos, ou seja, já tinham atingido a terceira idade, e os $41,4 \%$ restantes tinham entre 40 e 60 anos. Estes dados confirmam os achados na literatura os quais indicam que $o$ diabetes tipo 2 acomete indivíduos a partir da quarta década de vida (Lerário, 1998).
Percebeu-se, ainda, um número elevado de indivíduos com mais de 70 anos (34,5\%), fato citado por Patarra (1995) como um indicador de que a população tem apresentado uma maior expectativa de vida.

Com relação ao estado civil, 19 indivíduos $(65,5 \%)$ eram casados, $7(24,1 \%)$ viúvos e 3 $(10,4 \%)$ separados. Dever (1988) destaca a freqüente relação entre o estado civil e a morbidade e mortalidade, evidenciando o coeficiente mais elevado de mortalidade entre viúvos, divorciados e solteiros e mais baixo entre os casados.

Quanto ao grau de escolaridade, observaram-se dois indivíduos (6,9\%) analfabetos, $75,8 \%$ da amostra com o primeiro grau incompleto, $10,3 \%$ com o primeiro grau completo e apenas $6,9 \%$ com o segundo grau completo. Assim, esta amostra foi constituída por um elevado percentual de pessoas com baixa escolaridade, o que pode representar dificuldades no entendimento de orientações terapêuticas e, conseqüentemente, no seu seguimento.

A ocupação predominante foi o trabalho doméstico $(58,6 \%)$ : $31 \%$ da amostra foi constituída de aposentados, constatação já esperada devido à faixa etária de maior incidência (58,6\% acima de 60 anos). Apenas 3 indivíduos $(10 \%)$ relataram ainda exercer atividade ocupacional.

Quanto ao tempo do primeiro diagnóstico referido pelos portadores de diabetes, pode-se observar, que $86,2 \%$ conviviam com a doença havia menos de 20 anos e, destes, 51,7\% havia menos de 10 anos (Tabela 1).

Com referência ao local onde se deu o primeiro diagnóstico, a maioria aconteceu em nível ambulatorial, assim considerado evidenciado em consultas de nível básico, isto é, em postos de saúde, em ambulatórios existentes em escolas ou mesmo em clínicas particulares. Já o diagnóstico realizado no nível terciário de assistência, ou seja, em hospitais, representou uma pequena parcela (seis indivíduos) desta população e pode ter 
ocorrido durante exames de rotina pré-cirúrgica, com suspeita de o indivíduo ser um portador de diabetes ou independente dela.

Em relação às orientações fornecidas no momento do diagnóstico observa-se, (Tabela 2), que somente $17,2 \%$ receberam orientações quanto à dieta, ao exercício físico e à medicação, as quais são consideradas por vários autores como o ponto de partida para qualquer tratamento a indivíduos portadores de diabetes (Programa..., 1996; Chacra \& Lerário, 1998). Destaca-se a não inclusão da prática de atividade física nas demais categorias de recomendações recebidas, que somam $82,8 \%$. Além disso, $70 \%$ das orientações iniciais constituíam-se da utilização medicamentosa e somente $27 \%$ (8 indivíduos) foram orientados para mudança no plano alimentar antes de iniciar a medicação. Por outro lado 3,4\% da amostra relatou não ter recebido qualquer tipo de informação.

Estes relatos reforçam a ênfase dada na orientação baseada quase exclusivamente no tratamento medicamentoso. No entanto, não se pode atribuir o sucesso terapêutico apenas a esta prática, excluindo-se os resultados oriundos de outras terapias.

Ressalta-se também o fato de não ter havido qualquer referência a instruções sobre mudança de estilo de vida, como a adoção de um plano alimentar saudável e a prática de exercício físico para o controle glicêmico. A orientação para dieta e exercício, segundo a Sociedade Brasileira de Diabetes (Programa..., 1996), deveria ser a primeira conduta visando o tratamento do diabetes tipo 2, mas não foi mencionada e não está, portanto, relacionada nos tipos de recomendação recebida pelos indivíduos que compõem a amostra desta investigação.

A análise da categoria profissional responsável por este tipo de orientação no momento do diagnóstico de diabetes é preocupante, pois as respostas identificaram quase em sua totalidade $(96,5 \%)$ o médico. Somente em um caso $(3,4 \%)$ a informação recebida pelo

Tabela 1. Tempo e local do primeiro diagnóstico de Diabetes Mellitus de um grupo de indivíduos inscritos no Programa de Assistência ao Indivíduo Diabético em Ribeirão Preto, 1999.

\begin{tabular}{|c|c|c|c|c|c|c|}
\hline \multirow[b]{2}{*}{ Tempo } & \multicolumn{2}{|c|}{ Ambulatório } & \multicolumn{2}{|c|}{ Hospital } & \multicolumn{2}{|c|}{ Total } \\
\hline & $\mathrm{n}$ & $\%$ & $n$ & $\%$ & $n$ & $\%$ \\
\hline $1-10$ & 13 & 56,5 & 2 & 33,3 & 15 & 51,7 \\
\hline $10-20$ & 8 & 34,7 & 2 & 33,3 & 10 & 34,5 \\
\hline $20-30$ & 2 & 8,7 & 1 & 16,6 & 3 & 10,3 \\
\hline $30-40$ & - & - & 1 & 16,6 & 1 & 3,5 \\
\hline $30-40$ & - & - & 1 & 16,6 & 1 & 3,5 \\
\hline Total & 23 & 100,0 & 6 & 100,0 & 29 & 100,0 \\
\hline
\end{tabular}

Tabela 2. Tipos de orientação recebida durante o primeiro diagnóstico de Diabetes Mellitus por um grupo de indivíduos portadores da doença em 1999.

\begin{tabular}{|c|c|c|c|c|c|c|}
\hline \multirow[b]{2}{*}{ Orientação } & \multicolumn{2}{|c|}{ Ambulatório } & \multicolumn{2}{|c|}{ Hospital } & \multicolumn{2}{|c|}{ Total } \\
\hline & $n$ & $\%$ & $n$ & $\%$ & $n$ & $\%$ \\
\hline Dieta & 8 & 34,7 & - & - & 8 & 27,6 \\
\hline Dieta $+\mathrm{m}^{1}$ & 8 & 34,7 & 4 & 66,6 & 12 & 41,4 \\
\hline Dieta $+i^{2}$ & 1 & 4,3 & - & - & 1 & 3,4 \\
\hline$D+e^{3}+m$ & 4 & 17,4 & 1 & 16,6 & 5 & 17,2 \\
\hline Medicação & 1 & 4,3 & 1 & 16,6 & 2 & 6,9 \\
\hline Não recebeu & 1 & 4,3 & - & - & 1 & 3,4 \\
\hline Total & 23 & 100,0 & 6 & 100,0 & 29 & 100,0 \\
\hline
\end{tabular}

(1) Medicação via oral; ${ }^{(2)}$ Insulina; (3) Exercício físico. 
entrevistado foi de que a recomendação havia sido feita por um funcionário da área de enfermagem.

\section{O N CLUS ÃO E CONSIDERAÇÕES FINAIS}

Com base nos resultados obtidos com esta investigação, pode-se concluir que, para a amostra estudada, a orientação inicial para o tratamento de Diabetes Mellitus recebida foi inadequada e condicionada ao tratamento medicamentoso, contrariando as atuais recomendações para este tipo de doença (Programa..., 1996; Sociedade Brasileira..., 1997; Chacra \& Lerário, 1998), as quais buscam também a adição de outras práticas terapêuticas, como o exercício físico e o seguimento de um plano alimentar individualizado, resultando, muitas vezes, em mudança no estilo de vida.

Além disso, embora o diagnóstico dos indivíduos estudados tenha se dado em décadas passadas, já havia, nessa época, recomendações específicas para planos alimentares e uma tendência a para a valorização de mudanças no estilo de vida e para a adoção da prática de exercício físico (Powers, 1992).

Pode-se comprovar, em parte, a hipótese lançada nesta pesquisa com referência à supervalorização do tratamento medicamentoso. No tocante ao predomínio do seguimento de recomendações oriundas do profissional médico, questiona-se o quanto os demais integrantes da equipe de saúde, como o enfermeiro e o nutricionista, por exemplo, têm assumido o posto de membro efetivo e constante nos grupos de assistência ao portador de diabetes.

Entende-se que há, ainda, a necessidade de novos estudos com o objetivo de avaliar este questionamento e também a efetividade das demais práticas terapêuticas, além do tratamento medicamentoso, ou seja: é preciso conhecer melhor as considerações do indivíduo portador de diabetes em relação ao seu tratamento e saber qual a importância dada por ele à prática de atividades como o exercício físico e à adesão ao plano alimentar. Pode-se afirmar, por outro lado, que é necessário, também, um preparo mais específico de toda a equipe de saúde, ampliando seus conhecimentos e permitindo assim melhor adequação nas orientações a serem fornecidas aos pacientes com esta doença.

Há, portanto, uma crescente demanda de mobilização dos membros da equipe de saúde do Município de Ribeirão Preto para efetivar uma prática multiprofissional entre eles, de forma a estabelecerem um tratamento coerente com as atuais propostas de assistência ao paciente portador de diabetes, incrementando novas orientações às pessoas em geral, principalmente àqueles indivíduos acometidos por doenças crônicas, visando garantir melhor qualidade de vida à população.

REFERÊ NCIAS B I B L I O G R Á F I C A S

BANCO MUNDIAL. Brasil: novo desafio à saúde do adulto. Washington DC, 1991. Parte 1. 134p. (Séries de Estudos do Banco Mundial sobre Países; Mimeografado).

BOOG, M.C.F., MAGRINI, V.F.P.L. Relato de experiência: reeducação alimentar por meio de abordagem interdisciplinar envolvendo as áreas de Nutrição e Saúde Mental. Revista da Sociedade de Cardiologia do Estado de São Paulo, São Paulo, v.9, n.3, p.1-8, 1999. Suplemento A.

BRASIL. Ministério da Saúde. Doenças crônico-degenerativas: evolução e tendências atuais. Brasília, 1988. 46p.

BRASIL. Ministério da Saúde. Manual de diabetes. 2.ed. Brasília : Secretaria de Assistência à Saúde, 1993. 92p.

CHACRA, A.R., LERÁRIO, D.D.G. Novos avanços na terapia do diabetes do tipo 2. Revista da Sociedade de Cardiologia do Estado de São Paulo, São Paulo, v.8, n.5, p.914-922, 1998.

DEVER, D.E.A. A epidemiologia na administração dos serviços de saúde. São Paulo : Pioneira, 1988. 385p. Cap.1: Epidemiologia: enfocando a prevenção. 
EXPERT COMMITTEE ON THE DIAGNOSIS AND CLASSIFICATION OF DIABETES MELLITUS. Report of the Expert Committee on the diagnosis and classification of Diabetes Mellitus. Diabetes Care, New York, v.22, p.S5-S19, 1999. Supplement 1.

FOSS, M.C., PACCOLA, G.M.F., SOUZA, N.V., YAZIGI, N. Estudo analítico de uma amostra populacional de diabéticos tipo 2 de Ribeirão Preto (SP). Revista da Associação Médica Brasileira, Ribeirão Preto, v.35, n.5, p.179-183, 1989.

GORDON, N.F. Diabetes: seu manual completo de exercícios. São Paulo: Physis, 1997. 140p. Cap. 2: Benefícios trazidos pelo exercício físico aos portadores de diabetes.

HOETT, J.J. Uma esperança para os diabéticos. A Saúde do Mundo, p.4-5, maio-jun. 1991.

LERÁRIO, A.C. Diabetes mellitus: aspectos epidemiológicos. Revista da Sociedade de Cardiologia do Estado de São Paulo, São Paulo, v.5, n.5, p.885-891, 1998.

MALERBI, D.A. Estudo da prevalência do diabetes mellitus no Brasil. São Paulo, 1991. 154p. Tese (Doutorado) - Faculdade de Medicina, Universidade de São Paulo, 1991.

MENDES, I.J.M. Promoção de saúde: caminhando para o único. Ribeirão Preto, 1996. 163p. Tese (Livre-Docência) - Escola de Enfermagem de Ribeirão Preto, Universidade de São Paulo, 1996.

PATARRA, N.L. Mudanças na dinâmica demográfica. In: MONTEIRO, C.A. Velhos e novos males da saúde no Brasil: a evolução do país e de suas doenças. São Paulo : Hucitec, 1995. Cap.3.

POWERS, M.A. A review of recent events in the history of diabetes nutritional care. Diabetes Education, Minnesota, v.18, n.5, p.393-400, 1992.

PROGRAMA Harvard Joslin - SBD. Educação em diabetes no Brasil: aspectos fundamentais do diagnóstico e tratamento do diabetes mellitus. Brasília: SBD, 1996. 94p.

PUPO, A.A. Diabetes mellitus na criança e adolescente. In: SETIAN, N. (Coord.). Endocrinologia pediátrica: aspectos físicos e metabólicos do recém-nascido ao adolescente. São Paulo : Sarvier, 1989. p.173-210.

REGENGA, M.M., BOTELHO, A.P.V., COLLETTI, G.A. Alterações fisiológicas e adequação do exercício em pacientes diabéticos. Revista da Sociedade de Cardiologia do Estado de São Paulo, São Paulo, v.8, n.5, p.16-22, 1998. Suplemento A.

RIGSBY, I. Delineamento de pesquisa de levantamento. In: SELLTIZ, C., WRIGHTSMAN, L.S., COOK, S.W. Métodos de pesquisa nas relações sociais. 2.ed. São Paulo : EPU, 1987. v.1: p.49-56.

RULL, J.A., ZORRILLA, E., JADZINSKY, M.N., SANTIAGO, J.V. Diabetes mellitus: complicações crônicas. México : McGraw-Hill, 1992.

SÃO PAULO. Secretaria de Saúde. Centro de Apoio e Desenvolvimento das Ações Integradas de Saúde. Programa de educação e controle do diabetes mellitus no SUDS - SP. São Paulo, 1991. p.75.

SOCIEDADE BRASILEIRA DE DIABETES. Consenso Brasileiro de Conceitos e Condutas para o diabetes mellitus: recomendações da Sociedade Brasileira de Diabetes para a prática clínica. São Paulo, 1997. 56p.

SOCIEDADE BRASILEIRA DE DIABETES. Diagnóstico e classificação do diabetes mellitus e tratamento do diabetes mellitus tipo 2, 2000. Disponível em: $<$ http://www.diabetess.org.br>. Acesso em: 22 ago. 2000.

THURM, U., HARPER, P.N. I'm Running on Insulin. Diabetes Care, New York, v.15, p.1811-1813, 1992. Supplement 4.

Recebido para publicação em 7 de agosto de 2000 e aceito em 6 de abril de 2001. 